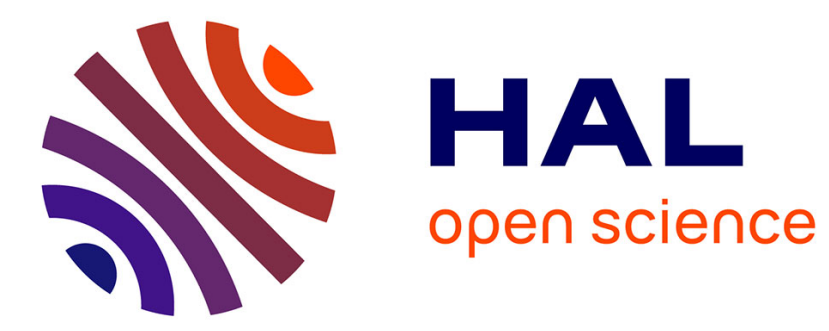

\title{
Two limit cases of Born-Infeld equations
}

Yue-Jun Peng, Jérémy Ruiz

\section{- To cite this version:}

Yue-Jun Peng, Jérémy Ruiz. Two limit cases of Born-Infeld equations. Journal of Hyperbolic Differential Equations, 2007, 4 (4), pp.565-586. hal-00488997

\section{HAL Id: hal-00488997 \\ https://hal.science/hal-00488997}

Submitted on 3 Jun 2010

HAL is a multi-disciplinary open access archive for the deposit and dissemination of scientific research documents, whether they are published or not. The documents may come from teaching and research institutions in France or abroad, or from public or private research centers.
L'archive ouverte pluridisciplinaire HAL, est destinée au dépôt et à la diffusion de documents scientifiques de niveau recherche, publiés ou non, émanant des établissements d'enseignement et de recherche français ou étrangers, des laboratoires publics ou privés. 


\title{
TWO LIMIT CASES OF BORN-INFELD EQUATIONS
}

\author{
YUE-JUN PENG AND JÉRÉMY RUIZ
}

Laboratoire de Mathématiques, CNRS UMR 6620

Université Blaise Pascal (Clermont-Ferrand 2), 63177 Aubière cedex, France

peng@math.univ-bpclermont.fr, ruiz@math.univ-bpclermont.fr

\begin{abstract}
We study two limit cases $\lambda \rightarrow \infty$ and $\lambda \rightarrow 0$ in Born-Infeld equations. Here the parameter $\lambda>0$ is interpreted as the maximal electric field in the electromagnetic theory and the case $\lambda=0$ corresponds to the string theory. Formal limits are governed by the classical Maxwell equations and pressureless magnetohydrodynamics system, respectively. For studying the limit $\lambda \rightarrow \infty$, a new scaling is introduced. We give the relations between these limits and Brenier high and low field limits. Finally, using compensated compactness arguments, the limits are rigorously justified for global entropy solutions in $L^{\infty}$ in one space dimension, based on derived uniform estimates and techniques for linear Lagrangian systems.
\end{abstract}

Keywords : Born-Infeld equations; high and low field limits; classical Maxwell equations; pressureless magnetohydrodynamics system.

Mathematics Subject Classification 2000 : 35B40, 35L60, 35Q35

\section{INTRODUCTION}

The Born-Infeld system is a nonlinear version of classical Maxwell equations. It was introduced by Born and Infeld in 1930's to describe physical phenomena of electromagnetism [4]. Recently, the Born-Infeld system has attracted considerable attention because of its new applications in the string theory and high energy physics. See Boillat [1], Brenier [5, 6] and Gibbons [12].

In the electromagnetic theory, $E$ and $B$ stand for the electric and magnetic fields in $\mathbb{R}^{3}$. The Born-Infeld system is defined through the Born-Infeld Lagrangian $L_{\lambda}$ and the Faraday law. The expression of $L_{\lambda}$ is (see [6]) :

$$
L_{\lambda}(B, E)=-\sqrt{\lambda^{2}+|B|^{2}-|E|^{2}-\frac{(B \cdot E)^{2}}{\lambda^{2}}},
$$

where "." stands for the inner product and $|\cdot|$ the Euclidean norm. The single parameter $\lambda>0$ can be interpreted as the maximal electric field.

Introduce the electric induction $D=\frac{\partial L_{\lambda}}{\partial E}$, the Poynting vector $P$ and the Born-Infeld energy density $h[4,5,13]$ :

$$
P=D \times B, \quad h=\sqrt{\lambda^{2}+|B|^{2}+\lambda^{2}|D|^{2}+|D \times B|^{2}} .
$$


Then

$$
E=\frac{\partial h(D, B)}{\partial D}=\frac{\lambda^{2} D+B \times P}{h}
$$

and the Born-Infeld equations, depending on the parameter $\lambda>0$, read :

$$
\begin{gathered}
\partial_{t} D+\nabla \times\left(\frac{-B+D \times P}{h}\right)=0, \\
\partial_{t} B+\nabla \times\left(\frac{\lambda^{2} D+B \times P}{h}\right)=0,
\end{gathered}
$$

The differential constraints are :

$$
\nabla \cdot D=\nabla \cdot B=0
$$

which are compatible with equations (1.4)-(1.5). Furthermore, $h$ and $P$ satisfy the additional conservation laws :

$$
\begin{gathered}
\partial_{t} h+\nabla \cdot P=0, \\
\partial_{t} P+\nabla \cdot\left(\frac{P \otimes P-B \otimes B-\lambda^{2} D \otimes D}{h}\right)-\nabla\left(\frac{\lambda^{2}}{h}\right)=0 .
\end{gathered}
$$

System (1.4)-(1.6) of 6 equations is abbreviated to $\lambda$-BI system. The case $\lambda=1$ has been intensively investigated. In [5], Brenier enlarged the 1-BI system to an augmented BornInfeld system of 10 equations by considering $h, D, B$ and $P$ as independent variables. He found many interesting properties of this last system, like fully linear degeneracy, Galilian invariance and existence of a strictly convex entropy which implies the hyperbolicity of the system in the sense of Friedrichs. Obviously, the augmentation of the $\lambda$-BI system can be carried out for any $\lambda>0$ in a same way. The resulting system (1.4)-(1.8) is referred to $\lambda$-ABI system. It is a hyperbolic system which admits a strictly convex entropy

$$
S_{\lambda}(D, B, P, h)=\frac{\lambda^{2}+|B|^{2}+\lambda^{2}|D|^{2}+|P|^{2}}{2 h}, \quad h>0 .
$$

Note that a different enlargement of the Born-Infeld system was proposed by Serre in [20], in which only $P$ is added as independent variable. Some extensions of the works $[5,20]$ to more general equations derived from the variational principle were given by Boillat [2].

For smooth solutions to the 1-BI and 1-ABI systems, the reader is referred to Brenier [5] and Serre [20] for local existence by an argument for first order symmetrizable hyperbolic systems, to Chae-Huh [10] for global existence with small initial data, and also to [7] for the solutions beyond singularities. Entropy solutions in one space dimension are studied in $[16,17]$ by the change of variables of Euler-Lagrange type (see [22]). For general theory of hyperbolic systems of conservation laws, we refer to $[19,11,9,14]$.

Following Brenier, (1.2) is called BI-manifold. It was shown that the BI-manifold of the 1-ABI system is time invariant for smooth solutions (see $[20,2,8]$ ) and also for entropy solutions in one space dimension (see $[16,17]$ ). The invariance of the BI-manifold implies the equivalence of the corresponding solutions between the 1-BI system and 1ABI system. As consequences, it yields the existence of local smooth solutions and global entropy solutions in one space dimension of the 1-BI system. Similar results hold for the $\lambda$-BI and $\lambda$-ABI systems for all $\lambda>0$. Thus, the enlargement of the $\lambda$-BI system provides a useful technique to study its Cauchy problem and asymptotic analysis. Indeed, 
it suffices to study the $\lambda$-ABI system and then take the initial data in the BI-manifold to get the corresponding results for the $\lambda$-BI system.

In this work, we are concerned with two limits $\lambda \rightarrow \infty$ and $\lambda \rightarrow 0$ in the $\lambda$-BI system. In classical Born-Infeld theory, $\lambda$ is a large parameter which controls the electric field, namely, $|E| \leq \lambda$. However, in Tachyonic condensate equations of high energy physics and the classical string theory, the corresponding value of $\lambda$ is zero (see $[6,12]$ ). Then it is important to consider both the limits $\lambda \rightarrow \infty$ and $\lambda \rightarrow 0$ in the $\lambda$-BI system to recover the link with the above physical situations.

This paper is organized as follows. In the next section, we first show that the Born-Infeld Lagrangian $L_{\lambda}$ is well defined for all $\lambda>0$. Then we perform formal asymptotic analysis in the $\lambda$-ABI system to recover the limit equations. They are governed by the classical Maxwell equations as $\lambda \rightarrow \infty$ and the pressureless magnetohydrodynamics system as $\lambda \rightarrow 0$. We show that these equations correspond to the limit cases of the Lagrangian and a new scaling is necessary to make the limit $\lambda \rightarrow \infty$ rigorously. Furthermore, we give the relations between these limits and Brenier high and low field limits in the 1-ABI system. From section 3, we consider the problem in one space dimension. We show the hyperbolic structure of the $\lambda$-ABI system for all $\lambda \geq 0$, which yields the existence of global entropy solutions of its Cauchy problem in $L^{\infty}$. These results are extensions of the corresponding results for the 1-ABI system. In particular, we give an explicit expression of the entropy solution and its dependence on $\lambda$. This yields uniform estimates for the sequences of the entropy solutions. The rigorous justification of the limits $\lambda \rightarrow \infty$ and $\lambda \rightarrow 0$ in the $\lambda$-ABI system is completed in section 4 and section 5 by using the compensated compactness arguments and techniques for linear Lagrangian systems. Finally, we remark that both convergence results hold globally in time because the limit systems are linearly hyperbolic.

\section{Preliminary}

2.1. Remarks on the definition of $L_{\lambda}$. In the literature there exist another expressions for the Born-Infeld Lagrangian and energy density. They are given by (see [2]) :

$$
\begin{gathered}
L_{k}(\widetilde{B}, \widetilde{E})=-\sqrt{k^{2}+k|\widetilde{B}|^{2}-k|\widetilde{E}|^{2}-(\widetilde{B} \cdot \widetilde{E})^{2}}, \\
h(\widetilde{D}, \widetilde{B})=\sqrt{k^{2}+k\left(|\widetilde{B}|^{2}+|\widetilde{D}|^{2}\right)+(\widetilde{D} \times \widetilde{B})^{2}},
\end{gathered}
$$

with $k \geq 0$ and

$$
\widetilde{E}=\frac{\partial h(\widetilde{D}, \widetilde{B})}{\partial \widetilde{D}}=\frac{k \widetilde{D}+\widetilde{B} \times \widetilde{P}}{h}, \quad \widetilde{P}=\widetilde{D} \times \widetilde{B}
$$

One may check that the relations between (1.1)-(1.2) and (2.1)-(2.2) are

$$
k=\lambda, \quad \widetilde{B}=\frac{B}{\sqrt{k}}, \quad \widetilde{D}=\sqrt{k} D .
$$

Moreover, both expressions $L_{\lambda}$ and $L_{k}$ are well defined for sufficiently large $\lambda$ and $k$, for instance, for $|E| \leq \lambda$ and $|\widetilde{E}| \leq k$, respectively. It is clear that $L_{k}$ is not well defined when $k=0$ unless $\widetilde{B} \cdot \widetilde{E}=0$. However, in the limit case $\lambda \rightarrow 0, L_{\lambda}$ corresponds to the Tachyonic condensate equations of high energy physics and the classical string theory (see [6]). Therefore, we should show that the Lagrangian $L_{\lambda}(B, E)$ given by $(1.1)$ is well 
defined for all $\lambda>0$. This fact is the advantage of the expression $L_{\lambda}$ compared to that of $L_{k}$. A more precise result can be stated as follows.

Proposition 2.1. For all $h>0$ and $\lambda>0$, we have

$$
L_{\lambda}(B, E)=-\frac{1}{h}\left(\lambda^{2}+|B|^{2}\right) .
$$

Proof. Set

$$
\Delta_{\lambda}(B, E)=\lambda^{2}+|B|^{2}-|E|^{2}-\frac{(B \cdot E)^{2}}{\lambda^{2}}
$$

with

$$
E=\frac{\lambda^{2} D+B \times P}{h}, \quad P=D \times B, \quad h=\sqrt{\lambda^{2}+|B|^{2}+\lambda^{2}|D|^{2}+|D \times B|^{2}} .
$$

Since

$$
B \cdot E=\frac{\lambda^{2}(B \cdot D)}{h}+\frac{B \cdot(B \times P)}{h}=\frac{\lambda^{2}(B \cdot D)}{h}
$$

and

$$
|E|^{2}=\frac{\left|\lambda^{2} D+B \times P\right|^{2}}{h^{2}}=\frac{1}{h^{2}}\left[\lambda^{4}|D|^{2}+|B \times P|^{2}+2 \lambda^{2} D \cdot(B \times P)\right],
$$

we get

$$
\begin{aligned}
\Delta_{\lambda}(B, E)= & \frac{1}{h^{2}}\left[\left(\lambda^{2}+|B|^{2}\right)\left(\lambda^{2}+|B|^{2}+\lambda^{2}|D|^{2}+|D \times B|^{2}\right)\right. \\
& \left.-\left(\lambda^{4}|D|^{2}+|B \times P|^{2}+2 \lambda^{2} D \cdot(B \times P)\right)-\lambda^{2}(D \cdot B)^{2}\right] \\
= & \frac{1}{h^{2}}\left[\left(\lambda^{2}+|B|^{2}\right)^{2}+\lambda^{2}\left(|D \times B|^{2}+|D|^{2}|B|^{2}-(D \cdot B)^{2}\right)\right. \\
& \left.+|B|^{2}|D \times B|^{2}-|B \times P|^{2}-2 \lambda^{2} D \cdot(B \times P)\right] .
\end{aligned}
$$

Using the relation

$$
|\xi|^{2}|\eta|^{2}=|\xi \cdot \eta|^{2}+(\xi \times \eta)^{2}, \quad \forall \xi, \eta \in \mathbb{R}^{3}
$$

we have

$$
\Delta_{\lambda}(B, E)=\frac{1}{h^{2}}\left[\left(\lambda^{2}+|B|^{2}\right)^{2}+2 \lambda^{2}|D \times B|^{2}+|B \cdot P|^{2}-2 \lambda^{2} D \cdot(B \times P)\right] .
$$

But,

$$
B \cdot P=B \cdot(D \times B)=0, \quad D \cdot(B \times P)=(D \times B) \cdot P=|D \times B|^{2} .
$$

Then

$$
\Delta_{\lambda}(B, E)=\frac{1}{h^{2}}\left(\lambda^{2}+|B|^{2}\right)^{2} \geq 0, \quad \forall \lambda>0 .
$$

This shows (2.3). 
2.2. Formal asymptotic analysis. As shown in the Introduction, the value of $\lambda$ is large in the electromagnetic theory and zero in the classical string theory. Then, it is important to study the limit cases $\lambda \rightarrow 0$ and $\lambda \rightarrow+\infty$ in the $\lambda$-ABI system. The detailed derivation of the formal asymptotic limits can be done as follows.

First, it is clear that the formal limit $\lambda \rightarrow 0$ in the $\lambda$-ABI system (1.4)-(1.8) is given by

$$
\left\{\begin{array}{l}
\partial_{t} B+\nabla \times\left(\frac{B \times P}{h}\right)=0 \\
\partial_{t} h+\nabla \cdot P=0 \\
\partial_{t} P+\nabla \cdot\left(\frac{P \otimes P-B \otimes B}{h}\right)=0 \\
\partial_{t} D+\nabla \times\left(\frac{-B+D \times P}{h}\right)=0 \\
\nabla \cdot B=\nabla \cdot D=0
\end{array}\right.
$$

In the limit of the $\lambda$-BI system (1.4)-(1.6), according to the definition (1.2)-(1.3), $(h, P, E)$ should be given by

$$
P=D \times B, \quad h=\sqrt{|B|^{2}+|D \times B|^{2}}, \quad E=\frac{B \times P}{h} .
$$

Then, from (2.3), it is easy to check that the Born-Infeld Lagrangian satisfies

$$
\lim _{\lambda \rightarrow 0} L_{\lambda}(B, E)=-\frac{|B|^{2}}{\sqrt{|B|^{2}+|D \times B|^{2}}} .
$$

A straightforward computation using (2.5) shows that

$$
\lim _{\lambda \rightarrow 0} L_{\lambda}(B, E)=-\sqrt{|B|^{2}-|E|^{2}},
$$

which was obtained in [6]. Here by (2.5), the conditions $|B|^{2}-|E|^{2} \geq 0$ and $B \cdot E=0$ are always satisfied. Note that the first three equations in $(2.4)$ for $(B, h, P)$, independent of the last one for $D$, are called pressureless magnetohydrodynamics (PMHD) system [5]. As we will see in section 5, in one space dimension the PMHD system is hyperbolic except some critical cases. This is different from the pressureless gas dynamics.

Now consider the limit $\lambda \rightarrow+\infty$. To this end, let $\alpha \in[0,1)$ be a constant and introduce the scaling

$$
B=\lambda^{\alpha} B_{\lambda}, \quad D=\lambda^{\alpha-1} D_{\lambda}
$$

Then from (1.2), we have

$$
\begin{gathered}
P=D \times B=\lambda^{2 \alpha-1} D_{\lambda} \times B_{\lambda}, \\
h=\lambda \sqrt{1+\varepsilon\left(\left|B_{\lambda}\right|^{2}+\left|D_{\lambda}\right|^{2}\right)+\varepsilon^{2}\left|D_{\lambda} \times B_{\lambda}\right|^{2}},
\end{gathered}
$$

where $\varepsilon=\lambda^{2(\alpha-1)} \rightarrow 0$. If we choose the scaling

$$
h=\lambda h_{\lambda}, \quad P=\lambda^{2 \alpha-1} P_{\lambda},
$$


the $\lambda$-ABI system (1.4)-(1.8) becomes:

$$
\left\{\begin{array}{l}
\partial_{t} D_{\lambda}+\nabla \times\left(\frac{-B_{\lambda}+\varepsilon D_{\lambda} \times P_{\lambda}}{h_{\lambda}}\right)=0 \\
\partial_{t} B_{\lambda}+\nabla \times\left(\frac{D_{\lambda}+\varepsilon B_{\lambda} \times P_{\lambda}}{h_{\lambda}}\right)=0 \\
\partial_{t} h_{\lambda}+\varepsilon \nabla \cdot P_{\lambda}=0, \\
\partial_{t} P_{\lambda}+\nabla \cdot\left(\frac{\varepsilon P_{\lambda} \otimes P_{\lambda}-B_{\lambda} \otimes B_{\lambda}-D_{\lambda} \otimes D_{\lambda}}{h_{\lambda}}\right)=\frac{1}{\varepsilon} \nabla\left(\frac{1}{h_{\lambda}}\right), \\
\nabla \cdot D_{\lambda}=\nabla \cdot B_{\lambda}=0
\end{array}\right.
$$

As $\varepsilon \rightarrow 0$, i.e. $\lambda \rightarrow \infty$, from (2.7)-(2.8), we should have formally $h_{\lambda} \rightarrow 1$. Then from (2.9) the formal limit $(\bar{B}, \bar{D})=\lim _{\lambda \rightarrow \infty}\left(B_{\lambda}, D_{\lambda}\right)$ satisfies the linear Maxwell equations :

$$
\left\{\begin{array}{l}
\partial_{t} \bar{B}+\nabla \times \bar{D}=0 \\
\partial_{t} \bar{D}-\nabla \times \bar{B}=0 \\
\nabla \cdot \bar{B}=\nabla \cdot \bar{D}=0
\end{array}\right.
$$

For the rest of the limit equations, we set $h_{1 \lambda}=\left(h_{\lambda}-1\right) / \varepsilon$. Since

$$
\frac{1}{h_{\lambda}}=\frac{1}{1+\varepsilon h_{1 \lambda}}=1-\varepsilon h_{1 \lambda}+O\left(\varepsilon^{2}\right)
$$

then

$$
\frac{1}{\varepsilon} \nabla\left(\frac{1}{h_{\lambda}}\right)=-\nabla h_{1 \lambda}+O(\varepsilon)
$$

From $h_{\lambda} \rightarrow 1$ and $P_{\lambda}=O(1)$, we have

$$
\nabla \cdot\left(\frac{\varepsilon P_{\lambda} \otimes P_{\lambda}}{h_{\lambda}}\right) \longrightarrow 0, \quad \text { as } \lambda \rightarrow+\infty
$$

Thus, using the equations for $h_{\lambda}$ and $P_{\lambda}$ in $(2.9)$, the formal limit $\left(\bar{h}_{1}, \bar{P}\right)=\lim _{\lambda \rightarrow \infty}\left(h_{1 \lambda}, P_{\lambda}\right)$ satisfies the linear hyperbolic system :

$$
\left\{\begin{array}{l}
\partial_{t} \bar{h}_{1}+\nabla \cdot \bar{P}=0, \\
\partial_{t} \bar{P}+\nabla \bar{h}_{1}=\nabla \cdot(\bar{B} \otimes \bar{B}+\bar{D} \otimes \bar{D}),
\end{array}\right.
$$

when $\bar{D}$ and $\bar{B}$ are solved by $(2.10)$.

Concerning the convergence of the Born-Infeld Lagrangian, we use (1.3), (2.6) and (2.8) to obtain

$$
E=\frac{\lambda^{2} \lambda^{\alpha-1} D_{\lambda}+\lambda^{\alpha} \lambda^{2 \alpha-1} B_{\lambda} \times P_{\lambda}}{\lambda h_{\lambda}}
$$

It follows that

$$
\frac{(B \cdot E)^{2}}{\lambda^{2}}=\lambda^{2(2 \alpha-1)}\left(B_{\lambda} \cdot D_{\lambda}\right)^{2}
$$

Writing

$$
E=\lambda^{\alpha}\left(D_{\lambda}+O(\varepsilon)\right)
$$


and noting that $2(2 \alpha-1)<2 \alpha$, from (1.1) we obtain

$$
\begin{aligned}
L_{\lambda}(B, E)+\lambda & =\lambda-\sqrt{\lambda^{2}+|B|^{2}-|E|^{2}-\lambda^{-2}(B \cdot E)^{2}} \\
& =\frac{|E|^{2}-|B|^{2}-\lambda^{-2}(B \cdot E)^{2}}{\lambda+\lambda \sqrt{1+\lambda^{-2}\left(|B|^{2}-|E|^{2}\right)-\lambda^{-4}(B \cdot E)^{2}}} \\
& =\frac{\lambda^{2 \alpha-1}\left(\left|D_{\lambda}\right|^{2}-\left|B_{\lambda}\right|^{2}+O(\varepsilon)\right)}{1+\sqrt{1+\varepsilon\left(\left|B_{\lambda}\right|^{2}-\left|D_{\lambda}\right|^{2}\right)+O\left(\varepsilon^{2}\right)}} \\
& =\lambda^{2 \alpha-1}\left(\frac{1}{2}\left(\left|D_{\lambda}\right|^{2}-\left|B_{\lambda}\right|^{2}\right)+O(\varepsilon)\right) .
\end{aligned}
$$

Thus, as $\lambda \rightarrow+\infty$, we have

$$
\lim _{\lambda \rightarrow \infty} \lambda^{1-2 \alpha}\left(L_{\lambda}(B, E)+\lambda\right)=\frac{1}{2}\left(|\bar{D}|^{2}-|\bar{B}|^{2}\right) .
$$

This shows that the classical Maxwell theory corresponds to the limit of the Lagrangian $\lambda^{1-2 \alpha}\left(L_{\lambda}(B, E)+\lambda\right)$. In particular, it corresponds to the limit of $L_{\lambda}(B, E)+\lambda$ when $\alpha=1 / 2$ and to the limit of $\lambda\left(L_{\lambda}(B, E)+\lambda\right)$ when $\alpha=0$. The latter case was given by Brenier (see [6]).

2.3. Relations with Brenier high and low field limits. In [5], Brenier introduced the high and low field limits in the 1-ABI system. The low field limit is defined by the scaling

$$
B=\tau B_{\tau}, D=\tau D_{\tau}, P=\tau^{2} P_{\tau}, h=h_{\tau}=1+O\left(\tau^{2}\right),
$$

where $(B, D, h, P)$ is a solution of the 1-ABI system and $\tau>0$ is an artificial small parameter. Put the expression (2.12) into the 1-ABI system and let $\tau \rightarrow 0$, he found that the formal limit of $\left(B_{\tau}, D_{\tau}\right)$ satisfies the linear Maxwell equations (2.10). It is easy to check that the low field limit corresponds to the limit $\lambda \rightarrow \infty$ in the $\lambda$-ABI system with the choice $\tau=\lambda^{\alpha-1}$. Indeed, with the above choice and $\varepsilon=\lambda^{2(\alpha-1)},\left(B_{\tau}, D_{\tau}, h_{\tau}, P_{\tau}\right)$ defined by (2.12) satisfies exactly the system (2.9).

Similarly, the high field limit is defined by the following scaling in the 1-ABI system :

$$
B=\tau B_{\tau}, D=D_{\tau}, P=\tau P_{\tau}, h=\tau h_{\tau},
$$

where $(B, D, h, P)$ is a solution of the 1-ABI system and $\tau>0$ is an artificial large parameter. Put the expression (2.13) into the 1-ABI system and let $\tau \rightarrow \infty$, then the formal limit of $\left(B_{\tau}, h_{\tau}, P_{\tau}\right)$ satisfies the PMHD system. We may check easily that this high field limit corresponds to the limit $\lambda \rightarrow 0$ in the $\lambda$-ABI system with the choice $\tau=\lambda^{-1}$.

The high and low field limits have been rigorously justified for smooth solutions, in a recent paper of Brenier and Yong [8], in which the physical regimes of these limits are explained. The convergence holds essentially locally in time for the high field limit. Whereas it is global in time for the low field limit because its limit equations are linear hyperbolic and admit global smooth solutions. Using the relations between the high and low field limits and those of $\lambda \rightarrow \infty$ and $\lambda \rightarrow 0$ discussed above, we may apply their results to yield the convergence of the limits $\lambda \rightarrow \infty$ and $\lambda \rightarrow 0$ for smooth solutions in the $\lambda$-ABI system. On the other hand, the results of this paper justify the high and 
low field limits for global entropy solutions in one space dimension. For simplify the presentation of the paper, we will not write these results in details.

\section{BORN-INFELD SYSTEMS IN ONE SPACE DIMENSION}

3.1. Hyperbolic structure of the $\lambda$-ABI system. From now on we consider the BornInfeld systems in one space dimension. As in [5], we denote $x=x_{1}$ and consider the problems independent of $x_{2}$ and $x_{3}$. Then $B_{1}$ and $D_{1}$ are constants from (1.4)-(1.6). It follows that the $\lambda$-BI system (1.4)-(1.6) and $\lambda$-ABI system (1.4)-(1.8) are reduced to

$$
\begin{aligned}
& \left\{\begin{array}{l}
\partial_{t} D_{2}+\partial_{x}\left(\frac{B_{3}+D_{2} P_{1}-D_{1} P_{2}}{h}\right)=0, \\
\partial_{t} D_{3}+\partial_{x}\left(\frac{-B_{2}+D_{3} P_{1}-D_{1} P_{3}}{h}\right)=0, \\
\partial_{t} B_{2}+\partial_{x}\left(\frac{-\lambda^{2} D_{3}+B_{2} P_{1}-B_{1} P_{2}}{h}\right)=0, \\
\partial_{t} B_{3}+\partial_{x}\left(\frac{\lambda^{2} D_{2}+B_{3} P_{1}-B_{1} P_{3}}{h}\right)=0, \\
P=D \times B, \quad h=\sqrt{\lambda^{2}+|B|^{2}+\lambda^{2}|D|^{2}+|D \times B|^{2}},
\end{array}\right. \\
& \left\{\begin{array}{l}
\partial_{t} h+\partial_{x} P_{1}=0, \\
\partial_{t} P_{1}+\partial_{x}\left(\frac{P_{1}^{2}-\left(\lambda^{2}+B_{1}^{2}+\lambda^{2} D_{1}^{2}\right)}{h}\right)=0 \\
\partial_{t} D_{2}+\partial_{x}\left(\frac{B_{3}+D_{2} P_{1}-D_{1} P_{2}}{h}\right)=0, \\
\partial_{t} D_{3}+\partial_{x}\left(\frac{-B_{2}+D_{3} P_{1}-D_{1} P_{3}}{h}\right)=0 \\
\partial_{t} B_{2}+\partial_{x}\left(\frac{-\lambda^{2} D_{3}+B_{2} P_{1}-B_{1} P_{2}}{h}\right)=0 \\
\partial_{t} B_{3}+\partial_{x}\left(\frac{\lambda^{2} D_{2}+B_{3} P_{1}-B_{1} P_{3}}{h}\right)=0 \\
\partial_{t} P_{2}+\partial_{x}\left(\frac{P_{1} P_{2}-\lambda^{2} D_{1} D_{2}-B_{1} B_{2}}{h}\right)=0 \\
\partial_{t} P_{3}+\partial_{x}\left(\frac{P_{1} P_{3}-\lambda^{2} D_{1} D_{3}-B_{1} B_{3}}{h}\right)=0
\end{array}\right.
\end{aligned}
$$

respectively. Here we have used the notations

$$
B=\left(B_{1}, B_{2}, B_{3}\right)^{t}, \quad D=\left(D_{1}, D_{2}, D_{3}\right)^{t}, \quad P=\left(P_{1}, P_{2}, P_{3}\right)^{t}
$$

Now let us rewrite the $\lambda$-ABI system (3.2) by introducing :

$$
P_{1}=h v_{2}, D_{2}=h v_{3}, D_{3}=h v_{4}, B_{2}=h v_{5}, B_{3}=h v_{6}, P_{2}=h v_{7}, P_{3}=h v_{8} .
$$


Then the $\lambda$-ABI system (3.2) becomes :

$$
\left\{\begin{array}{l}
\partial_{t} h+\partial_{x}\left(h v_{2}\right)=0 \\
\partial_{t}\left(h v_{2}\right)+\partial_{x}\left(h v_{2}^{2}-a^{2} h^{-1}\right)=0 \\
\partial_{t}\left(h v_{3}\right)+\partial_{x}\left(h v_{2} v_{3}-D_{1} v_{7}+v_{6}\right)=0 \\
\partial_{t}\left(h v_{4}\right)+\partial_{x}\left(h v_{2} v_{4}-D_{1} v_{8}-v_{5}\right)=0 \\
\partial_{t}\left(h v_{5}\right)+\partial_{x}\left(h v_{2} v_{5}-B_{1} v_{7}-\lambda^{2} v_{4}\right)=0 \\
\partial_{t}\left(h v_{6}\right)+\partial_{x}\left(h v_{2} v_{6}-B_{1} v_{8}+\lambda^{2} v_{3}\right)=0 \\
\partial_{t}\left(h v_{7}\right)+\partial_{x}\left(h v_{2} v_{7}-\lambda^{2} D_{1} v_{3}-B_{1} v_{5}\right)=0 \\
\partial_{t}\left(h v_{8}\right)+\partial_{x}\left(h v_{2} v_{8}-\lambda^{2} D_{1} v_{4}-B_{1} v_{6}\right)=0
\end{array}\right.
$$

where the constant $a$, depending on $\lambda$, is defined by

$$
a=\sqrt{\lambda^{2}+B_{1}^{2}+\lambda^{2} D_{1}^{2}}
$$

Remark that the first two equations in (3.3) form the system for Chaplygin gas dynamics on which some results are obtained in $[18,15,5]$.

Suppose $h, v_{2} \in L^{\infty}\left(\mathbb{R}^{+} \times \mathbb{R}\right)$ with $h>0$. Using the first equations in (3.3), we may introduce a change of variables $(t, x) \longmapsto(s, y)$ from $\mathbb{R}^{+} \times \mathbb{R}$ to $\mathbb{R}^{+} \times \mathbb{R}$, with (see $[22,17]$ )

$$
s=t, \quad d y=h d x-h v_{2} d t .
$$

For simplicity, denote by $v(s, y)=v(t, x)$. Then in Lagrangian coordinates $(s, y)$, the system (3.3) is written as :

$$
\partial_{s} v+A \partial_{y} v=0, \quad t>0, \quad y \in \mathbb{R}
$$

where $v=\left(h^{-1}, v_{2}, \ldots, v_{8}\right)^{t}$ and

$$
A=\left(\begin{array}{cccccccc}
0 & -1 & 0 & 0 & 0 & 0 & 0 & 0 \\
-a^{2} & 0 & 0 & 0 & 0 & 0 & 0 & 0 \\
0 & 0 & 0 & 0 & 0 & 1 & -D_{1} & 0 \\
0 & 0 & 0 & 0 & -1 & 0 & 0 & -D_{1} \\
0 & 0 & 0 & -\lambda^{2} & 0 & 0 & -B_{1} & 0 \\
0 & 0 & \lambda^{2} & 0 & 0 & 0 & 0 & -B_{1} \\
0 & 0 & -\lambda^{2} D_{1} & 0 & -B_{1} & 0 & 0 & 0 \\
0 & 0 & 0 & -\lambda^{2} D_{1} & 0 & -B_{1} & 0 & 0
\end{array}\right)
$$

Recall that a linear Lagrangian system is a linear system in Lagrangian coordinates via a transformation of Euler-Lagrangian type [17]. Since $A$ is a constant matrix, the $\lambda$-ABI system (3.3) is a linear Lagrangian system and the results in [17] can be applied. A straightforward computation gives the eigenvalues of $A$ :

$$
\widetilde{\mu}_{1}=\widetilde{\mu}_{2}=\widetilde{\mu}_{3}=-a, \quad \widetilde{\mu}_{4}=\widetilde{\mu}_{5}=0, \quad \widetilde{\mu}_{6}=\widetilde{\mu}_{7}=\widetilde{\mu}_{8}=a .
$$

Moreover, set

$$
\beta=B_{1}^{2}+\lambda^{2} D_{1}^{2}, \quad \phi_{ \pm}=a D_{1} \pm B_{1}, \quad \psi_{ \pm}=a B_{1} \pm \lambda^{2} D_{1}
$$


For $\beta \neq 0$ (then $a \neq 0$ ) we choose the left eigenvectors $l_{i \lambda}$ and right eigenvectors $r_{j \lambda}$ of $A$ as follows :

$$
\begin{aligned}
\left\{\begin{aligned}
l_{1 \lambda} & =\left(0,0, \lambda^{2} \phi_{-}, \lambda^{2} \phi_{+}, \psi_{+}, \psi_{-}, \beta, \beta\right), \\
l_{2 \lambda} & =\left(0,0, \lambda^{2} \phi_{+},-\lambda^{2} \phi_{-}, \psi_{-},-\psi_{+}, \beta,-\beta\right), \\
l_{3 \lambda} & =(a, 1,0,0,0,0,0,0), \\
l_{4 \lambda} & =\frac{1}{2 a^{2}}\left(0,0, B_{1},-B_{1},-D_{1}, D_{1}, 1,1\right), \\
l_{5 \lambda} & =\frac{1}{2 a^{2}}\left(0,0,-B_{1},-B_{1}, D_{1}, D_{1}, 1,-1\right), \\
l_{6 \lambda} & =\left(0,0, \lambda^{2} \phi_{+}, \lambda^{2} \phi_{-}, \psi_{-}, \psi_{+},-\beta,-\beta\right), \\
l_{7 \lambda} & =\left(0,0, \lambda^{2} \phi_{-},-\lambda^{2} \phi_{+}, \psi_{+},-\psi_{-},-\beta, \beta\right), \\
l_{8 \lambda} & =(a,-1,0,0,0,0,0,0), \\
r_{1 \lambda} & =\frac{1}{4 a^{2} \beta}\left(0,0, \phi_{-}, \phi_{+}, \psi_{+}, \psi_{-}, \beta, \beta\right)^{t}, \\
r_{2 \lambda} & =\frac{1}{4 a^{2} \beta}\left(0,0, \phi_{+},-\phi_{-}, \psi_{-},-\psi_{+}, \beta,-\beta\right)^{t}, \\
r_{3 \lambda} & =\frac{1}{2 a}(1, a, 0,0,0,0,0,0)^{t}, \\
r_{4 \lambda} & =\left(0,0, B_{1},-B_{1},-\lambda^{2} D_{1}, \lambda^{2} D_{1}, \lambda^{2}, \lambda^{2}\right)^{t}, \\
r_{5 \lambda} & =\left(0,0,-B_{1},-B_{1}, \lambda^{2} D_{1}, \lambda^{2} D_{1}, \lambda^{2},-\lambda^{2}\right)^{t}, \\
r_{6 \lambda} & =\frac{1}{4 a^{2} \beta}\left(0,0, \phi_{+}, \phi_{-}, \psi_{-}, \psi_{+},-\beta,-\beta\right)^{t}, \\
r_{7 \lambda} & =\frac{1}{4 a^{2} \beta}\left(0,0, \phi_{-},-\phi_{+}, \psi_{+},-\psi_{-},-\beta, \beta\right)^{t}, \\
r_{8 \lambda} & =\frac{1}{2 a}(1,-a, 0,0,0,0,0,0)^{t} .
\end{aligned}\right.
\end{aligned}
$$

We see that $l_{i \lambda}$ and $r_{j \lambda}$ are defined for all $\lambda \geq 0$ and satisfy $l_{i \lambda} r_{j \lambda}=\delta_{i j}$ for all $1 \leq i, j \leq 8$. Hence, the matrix $A$ is diagonalizable and the linear system (3.4) is hyperbolic. When $\beta=0$ and $a \neq 0$, i.e., $B_{1}=D_{1}=0$ and $\lambda \neq 0$, we may write $A=\operatorname{diag}\left(A_{1}, A_{2}, 0\right)$, with

$$
A_{1}=\left(\begin{array}{cc}
0 & -1 \\
-a^{2} & 0
\end{array}\right), \quad A_{2}=\left(\begin{array}{cccc}
0 & 0 & 0 & 1 \\
0 & 0 & -1 & 0 \\
0 & -\lambda^{2} & 0 & 0 \\
\lambda^{2} & 0 & 0 & 0
\end{array}\right) \text {. }
$$

It is easy to check that $A_{1}$ and $A_{2}$ are diagonalizable. So is $A$ and then the $\lambda$-ABI system is hyperbolic for all $a \neq 0$ and all $\lambda \geq 0$.

The above computations are elementary but the expressions of $l_{i \lambda}$ and $r_{j \lambda}$ are important in the study of the limit $\lambda \rightarrow 0$. That is why we keep the subscript $\lambda$ in their expressions to see uniform estimates with respect to $\lambda$.

Now applying the results in [17], we have

Proposition 3.1. Let $B_{1}, D_{1} \in \mathbb{R}$ and $h>0$. Assume $a \neq 0$. Then the $\lambda$-ABI system (3.3) is a linear Lagrangian one and hyperbolic for all $\lambda \geq 0$. It admits a complete set 
of classical Riemann invariants $w_{i}=l_{i \lambda} v(1 \leq i \leq 8)$. The eigenvalues $\mu_{i}=\mu_{i}(v)$ $(1 \leq i \leq 8)$ of the system (3.3) are :

$$
\mu_{1}=\mu_{2}=\mu_{3}=v_{2}-a h^{-1}, \mu_{4}=\mu_{5}=v_{2}, \mu_{6}=\mu_{7}=\mu_{8}=v_{2}+a h^{-1} .
$$

Moreover, all entropy-entropy flux pairs $(E, F)$ of the system are expressed as

$$
(E(v), F(v))=h \sum_{i=1}^{8}\left(1, \mu_{i}(v)\right) g_{i}\left(l_{i \lambda} v\right),
$$

with arbitrary continuous functions $g_{i}(1 \leq i \leq 8)$.

Remark 3.1. When $a=0$, the system for Chaplygin gas dynamics becomes the pressureless gas dynamics which is not hyperbolic. This means that $a \neq 0$ is also a necessary condition to keep the hyperbolicity of the $\lambda$-ABI system.

3.2. Entropy solutions of the Born-Infeld systems. Consider the Cauchy problem to the $\lambda$-ABI system (3.3) associated to the initial conditions :

$$
t=0: \quad h=h^{0}(x), \quad v_{i}=v_{i}^{0}(x) \quad(2 \leq i \leq 8), \quad x \in \mathbb{R} .
$$

Following [15], in order to guarantee the strict hyperbolicity of the system for Chaplygin gas for all time $t \geq 0$ we need to assume that

$$
h^{0}, v_{i}^{0} \in L^{\infty}(\mathbb{R}) \quad(2 \leq i \leq 8), \quad \inf _{x \in \mathbb{R}}\left(v_{2}^{0}(x)+\frac{a}{h^{0}(x)}\right)>\sup _{x \in \mathbb{R}}\left(v_{2}^{0}(x)-\frac{a}{h^{0}(x)}\right),
$$

which imply that $h(t, x)>0$ for almost all $(t, x) \in \mathbb{R}^{+} \times \mathbb{R}$. Note that conditions (3.10) appeared first time in [18] in the study of BV weak solutions and their oscillations.

The Cauchy problems to the 1-ABI system and 1-BI system have been studied in $[16,17]$ through an Euler-Lagrange change of variables. It was shown that the Cauchy problems to these two systems admit each a unique entropy solution with an explicit expression. Since the $\lambda$-ABI system is hyperbolic linear Lagrange system, the mentioned results can be extended easily to the $\lambda$-ABI and $\lambda$-BI systems.

Recall that by entropy solution of the $\lambda$-ABI system we mean weak solution satisfying the entropy equality $\partial_{t} E(v)+\partial_{x} F(v)=0$ for all entropy-entropy flux pairs given in (3.8). Now let us define

$$
\begin{gathered}
Y^{0}(x)=\int_{0}^{x} h^{0}(\xi) d \xi \\
X(t, y)=\frac{1}{2} \int_{0}^{y+t}\left(v_{2}^{0}+\frac{a}{h^{0}}\right)\left(X^{0}(\xi)\right) d \xi-\frac{1}{2} \int_{0}^{y-t}\left(v_{2}^{0}-\frac{a}{h^{0}}\right)\left(X^{0}(\xi)\right) d \xi .
\end{gathered}
$$

It was shown that $Y^{0}$ and $y \longmapsto X(t, y)$ are Lipschitzian and bijective for all $t \geq 0$. We denote their inverse functions by

$$
X^{0}=\left(Y^{0}\right)^{-1}, \quad Y(t, \cdot)=X^{-1}(t, \cdot) .
$$

The results of the existence of entropy solutions to the $\lambda$-ABI system can be stated as follows. Their proofs will be omitted since they are similar to the cases for 1-ABI system [17]. 
Proposition 3.2. Let the assumption (3.10) hold and $a \neq 0$. Then the Cauchy problem (3.3) and (3.9) for the $\lambda$-ABI system admits a unique entropy solution $h, v \in L^{\infty}\left(\mathbb{R}^{+} \times \mathbb{R}\right)$. The solution satisfies the maximum principle:

$$
\begin{aligned}
& \inf _{\xi \in \mathbb{R}}\left(v_{2}^{0}(\xi)+\frac{a}{h^{0}(\xi)}\right) \leq v_{2}(t, x)+\frac{a}{h(t, x)} \leq \sup _{\xi \in \mathbb{R}}\left(v_{2}^{0}(\xi)+\frac{a}{h^{0}(\xi)}\right), \\
& \inf _{\xi \in \mathbb{R}}\left(v_{2}^{0}(\xi)-\frac{a}{h^{0}(\xi)}\right) \leq v_{2}(t, x)-\frac{a}{h(t, x)} \leq \sup _{\xi \in \mathbb{R}}\left(v_{2}^{0}(\xi)-\frac{a}{h^{0}(\xi)}\right),
\end{aligned}
$$

and has an explicit expression:

$$
v(t, x)=\sum_{i=1}^{8}\left(l_{i \lambda} v^{0}\right)\left(X^{0}\left(Y(t, x)-\widetilde{\mu}_{i} t\right)\right) r_{i \lambda},
$$

where $v^{0}=\left(\left(h^{0}\right)^{-1}, v_{2}^{0}, \cdots, v_{8}^{0}\right)^{t}, \widetilde{\mu}_{i}, l_{i \lambda}$ and $r_{i \lambda}$ are defined in (3.5)-(3.7), respectively.

Remark that the estimates (3.14)-(3.15) depend only on the system of Chaplygin gas dynamics and independent of the rest equations in the $\lambda$-ABI system. They are consequences of the fact that the system of Chaplygin gas dynamics is fully linearly degenerate. Applying Proposition 3.2 together with the time invariance of the BI-manifold proved in [17] yields a result of the existence and uniqueness of entropy solutions to the $\lambda$-BI system.

Proposition 3.3. Let $\left(B_{2}^{0}, B_{3}^{0}, D_{2}^{0}, D_{3}^{0}\right) \in L^{\infty}(\mathbb{R})$ and $\left(h^{0}, P^{0}\right)$ be defined by the $B I$ manifold (1.2) satisfying the second condition in (3.10). Assume $a \neq 0$. Then there exists a unique entropy solution $\left(B_{2}, B_{3}, D_{2}, D_{3}\right) \in L^{\infty}\left(\mathbb{R}^{+} \times \mathbb{R}\right)$ to the $\lambda$-BI system (3.1) with the initial data $\left(B_{2}^{0}, B_{3}^{0}, D_{2}^{0}, D_{3}^{0}\right)$.

\section{Limit toward LiNEAR MAXWELl EQUATIONS IN ONE DIMENSION}

4.1. Uniform estimates of the entropy solutions. In this section, we study the asymptotic limit $\lambda \rightarrow \infty$ in the $\lambda$-ABI system. We want to prove the convergence of the system (2.9) to the system (2.10)-(2.11) in one space dimension. First, in one space dimension the system $(2.9)$ reads :

$$
\left\{\begin{array}{l}
\partial_{t} h_{\lambda}+\partial_{x}\left(\varepsilon P_{1 \lambda}\right)=0 \\
\partial_{t}\left(\varepsilon P_{1 \lambda}\right)+\partial_{x}\left(\frac{\varepsilon^{2} P_{1 \lambda}^{2}-\rho_{\lambda}^{2}}{h_{\lambda}}\right)=0 \\
\partial_{t} D_{2 \lambda}+\partial_{x}\left(\frac{B_{3 \lambda}+\varepsilon\left(D_{2 \lambda} P_{1 \lambda}-D_{1} P_{2 \lambda}\right)}{h_{\lambda}}\right)=0 \\
\partial_{t} D_{3 \lambda}+\partial_{x}\left(\frac{-B_{2 \lambda}+\varepsilon\left(D_{3 \lambda} P_{1 \lambda}-D_{1} P_{3 \lambda}\right)}{h_{\lambda}}\right)=0 \\
\partial_{t} B_{2 \lambda}+\partial_{x}\left(\frac{-D_{3 \lambda}+\varepsilon\left(B_{2 \lambda} P_{1 \lambda}-B_{1} P_{2 \lambda}\right)}{h_{\lambda}}\right)=0 \\
\partial_{t} B_{3 \lambda}+\partial_{x}\left(\frac{D_{2 \lambda}+\varepsilon\left(B_{3 \lambda} P_{1 \lambda}-B_{1} P_{3 \lambda}\right)}{h_{\lambda}}\right)=0 \\
\partial_{t} P_{2 \lambda}+\partial_{x}\left(\frac{\varepsilon P_{1 \lambda} P_{2 \lambda}-D_{1} D_{2 \lambda}-B_{1} B_{2 \lambda}}{h_{\lambda}}\right)=0 \\
\partial_{t} P_{3 \lambda}+\partial_{x}\left(\frac{\varepsilon P_{1 \lambda} P_{3 \lambda}-D_{1} D_{3 \lambda}-B_{1} B_{3 \lambda}}{h_{\lambda}}\right)=0
\end{array}\right.
$$


where

$$
\varepsilon=\lambda^{2(\alpha-1)}, \quad \alpha \in[0,1), \quad \rho_{\lambda}=\sqrt{1+\varepsilon \sigma}, \quad \sigma=B_{1}^{2}+D_{1}^{2},
$$

with $B_{1}, D_{1}$ being two real constants. A more general case for which $\left(B_{1 \lambda}\right)_{\lambda}$ and $\left(D_{1 \lambda}\right)_{\lambda}$ are bounded sequences in $\mathbb{R}$ (then admitting convergent subsequences) can be dealt with in a similar way.

Let us recall the definition :

$$
\left\{\begin{array}{l}
P_{1 \lambda}=h_{\lambda} v_{2 \lambda}, D_{2 \lambda}=h_{\lambda} v_{3 \lambda}, D_{3 \lambda}=h_{\lambda} v_{4 \lambda}, B_{2 \lambda}=h_{\lambda} v_{5 \lambda}, \\
B_{3 \lambda}=h_{\lambda} v_{6 \lambda}, P_{2 \lambda}=h_{\lambda} v_{7 \lambda}, P_{3}=h_{\lambda} v_{8 \lambda} .
\end{array}\right.
$$

According to the scaling (2.7)-(2.8), we associate to (4.1) the following initial conditions :

$$
t=0: \quad h_{\lambda}=h_{\lambda}^{0}(x) \stackrel{\text { def }}{=} 1+\varepsilon h_{1 \lambda}^{0}(x), \quad v_{i \lambda}=v_{i \lambda}^{0}(x) \quad(2 \leq i \leq 8), \quad x \in \mathbb{R} .
$$

Observe that the first two equations in (4.1) are decoupled with the rest. Then uniform estimates for the variables $\left(h_{\lambda}, P_{1 \lambda}\right)$ and $\left(D_{2 \lambda}, D_{3 \lambda}, B_{2 \lambda}, B_{3 \lambda}, P_{2 \lambda}, P_{3 \lambda}\right)$ can be treated separately.

Lemma 4.1. Assume that the sequences of the initial data $\left(h_{1 \lambda}^{0}\right)_{\lambda},\left(v_{i \lambda}^{0}\right)_{\lambda}(2 \leq i \leq 8)$ are bounded in $L^{\infty}(\mathbb{R})$. Then the Cauchy problem (4.1)-(4.3) has a unique entropy solution $\left(h_{\lambda}, P_{1 \lambda}, D_{2 \lambda}, D_{3 \lambda}, B_{2 \lambda}, B_{3 \lambda}, P_{2 \lambda}, P_{3 \lambda}\right)$, and for sufficiently large $\lambda>0$, we have

$$
\left\|h_{\lambda}-1\right\|_{L^{\infty}\left(\mathbb{R}^{+} \times \mathbb{R}\right)} \leq C \varepsilon, \quad\left\|P_{1 \lambda}\right\|_{L^{\infty}\left(\mathbb{R}^{+} \times \mathbb{R}\right)} \leq C,
$$

where $C>0$ is a constant independent of $\lambda$.

Proof. Let us notice that under the assumption of Lemma 4.1, Proposition 3.2 can be applied to the Cauchy problem (4.1) and (4.3). Indeed, the hyperbolicity of the system is invariant under the change of variables (2.6) and (2.8). Moreover, for sufficiently large $\lambda$, we have

$$
\rho_{\lambda}=1+O(\varepsilon), \quad h_{\lambda}(0, x)=1+\varepsilon h_{1 \lambda}^{0}(x),
$$

which yield $\rho_{\lambda}>0$,

$$
\underline{w}_{\lambda} \stackrel{\text { def }}{=} \inf _{\xi \in \mathbb{R}}\left(\varepsilon v_{2 \lambda}^{0}(\xi)+\frac{\rho_{\lambda}}{1+\varepsilon h_{1 \lambda}^{0}(\xi)}\right)=1+O(\varepsilon)
$$

and

$$
\bar{z}_{\lambda} \stackrel{\text { def }}{=} \sup _{\xi \in \mathbb{R}}\left(\varepsilon v_{2 \lambda}^{0}(\xi)-\frac{\rho_{\lambda}}{1+\varepsilon h_{1 \lambda}^{0}(\xi)}\right)=-1+O(\varepsilon) .
$$

Thus, the condition (3.10) is trivially fulfilled and there is a unique entropy solution to the Cauchy problem (4.1) and (4.3).

Similarly,

$$
\begin{gathered}
\bar{w}_{\lambda} \stackrel{\text { def }}{=} \sup _{\xi \in \mathbb{R}}\left(\varepsilon v_{2 \lambda}^{0}(\xi)+\frac{\rho_{\lambda}}{1+\varepsilon h_{1 \lambda}^{0}(\xi)}\right)=1+O(\varepsilon), \\
\underline{z}_{\lambda} \stackrel{\text { def }}{=} \inf _{\xi \in \mathbb{R}}\left(\varepsilon v_{2 \lambda}^{0}(\xi)-\frac{\rho_{\lambda}}{1+\varepsilon h_{1 \lambda}^{0}(\xi)}\right)=-1+O(\varepsilon) .
\end{gathered}
$$

Applying the maximum principle (3.14)-(3.15) to the solution, we obtain

$$
\begin{gathered}
\underline{w}_{\lambda} \leq \varepsilon v_{2 \lambda}(t, x)+\frac{\rho_{\lambda}}{h_{\lambda}(t, x)} \leq \bar{w}_{\lambda}, \\
\underline{z}_{\lambda} \leq \varepsilon v_{2 \lambda}(t, x)-\frac{\rho_{\lambda}}{h_{\lambda}(t, x)} \leq \bar{z}_{\lambda} .
\end{gathered}
$$


It follows that $h_{\lambda}(t, x)=1+O(\varepsilon)$ and the sequence $\left(v_{2 \lambda}\right)_{\lambda}$ is bounded in $L^{\infty}\left(\mathbb{R}^{+} \times \mathbb{R}\right)$, which implies that the sequence $\left(P_{1 \lambda}\right)_{\lambda}$ is bounded in $L^{\infty}\left(\mathbb{R}^{+} \times \mathbb{R}\right)$.

Lemma 4.2. Under the assumptions of Lemma 4.1, the sequence $\left(D_{2 \lambda}, D_{3 \lambda}, B_{2 \lambda}, B_{3 \lambda}, P_{2 \lambda}, P_{3 \lambda}\right)_{\lambda}$ is bounded in $L^{\infty}\left(\mathbb{R}^{+} \times \mathbb{R}\right)$.

Proof. Since $h_{\lambda}>0$ for all $\lambda>0$ large, by the first equation in (4.1), we may make the change of variables : $(t, x) \longmapsto(s, y)$ with

$$
s=t, \quad d y=h_{\lambda} d x-\varepsilon P_{1 \lambda} d t .
$$

Then in Lagrangian coordinates $(s, y), \widetilde{v}_{\lambda}(s, y)=\left(v_{3 \lambda}, \cdots, v_{8 \lambda}\right)^{t}(t, x)$ satisfies an equivalent system

$$
\partial_{s} \widetilde{v}_{\lambda}+\widetilde{A}_{\lambda} \partial_{y} \widetilde{v}_{\lambda}=0
$$

where

$$
\widetilde{A}_{\lambda}=\left(\begin{array}{cccccc}
0 & 0 & 0 & 1 & -\varepsilon D_{1} & 0 \\
0 & 0 & -1 & 0 & 0 & -\varepsilon D_{1} \\
0 & -1 & 0 & 0 & -\varepsilon B_{1} & 0 \\
1 & 0 & 0 & 0 & 0 & -\varepsilon B_{1} \\
-D_{1} & 0 & -B_{1} & 0 & 0 & 0 \\
0 & -D_{1} & 0 & -B_{1} & 0 & 0
\end{array}\right)
$$

A straightforward computation shows that

$$
\nu_{1}=\nu_{2}=-\rho_{\lambda}, \quad \nu_{3}=\nu_{4}=0, \quad \nu_{5}=\nu_{6}=\rho_{\lambda}
$$

are the eigenvalues of $\widetilde{A}_{\lambda}$. For $\sigma \neq 0$, i.e., $B_{1}^{2}+D_{1}^{2} \neq 0$, we choose the corresponding left eigenvectors $\widetilde{l}_{i \lambda}$ and right eigenvectors $\widetilde{r}_{i \lambda}$ of $\widetilde{A}_{\lambda}$ as follows :

$$
\left\{\begin{array}{l}
\widetilde{l}_{1 \lambda}=\frac{1}{2}\left(\rho_{\lambda} D_{1}-B_{1}, \rho_{\lambda} D_{1}+B_{1}, \rho_{\lambda} B_{1}+D_{1}, \rho_{\lambda} B_{1}-D_{1}, \varepsilon \sigma, \varepsilon \sigma\right), \\
\widetilde{l}_{2 \lambda}=\frac{1}{2}\left(\rho_{\lambda} D_{1}+B_{1},-\rho_{\lambda} D_{1}+B_{1}, \rho_{\lambda} B_{1}-D_{1},-\left(\rho_{\lambda} B_{1}+D_{1}\right), \varepsilon \sigma,-\varepsilon \sigma\right), \\
\widetilde{l}_{3 \lambda}=\frac{1}{2 \rho_{\lambda}^{2}}\left(B_{1},-B_{1},-D_{1}, D_{1}, 1,1\right), \\
\widetilde{l}_{4 \lambda}=\frac{1}{2 \rho_{\lambda}^{2}}\left(-B_{1},-B_{1}, D_{1}, D_{1}, 1,-1\right), \\
\widetilde{l}_{5 \lambda}=\frac{1}{2}\left(\rho_{\lambda} D_{1}+B_{1}, \rho_{\lambda} D_{1}-B_{1}, \rho_{\lambda} B_{1}-D_{1}, \rho_{\lambda} B_{1}+D_{1},-\varepsilon \sigma,-\varepsilon \sigma\right), \\
\widetilde{l}_{6 \lambda}=\frac{1}{2}\left(\rho_{\lambda} D_{1}-B_{1},-\left(\rho_{\lambda} D_{1}+B_{1}\right), \rho_{\lambda} B_{1}+D_{1},-\rho_{\lambda} B_{1}+D_{1},-\varepsilon \sigma, \varepsilon \sigma\right),
\end{array}\right.
$$




$$
\left\{\begin{aligned}
\widetilde{r}_{1 \lambda} & =\frac{1}{2 \rho_{\lambda}^{2} \sigma}\left(\rho_{\lambda} D_{1}-B_{1}, \rho_{\lambda} D_{1}+B_{1}, \rho_{\lambda} B_{1}+D_{1}, \rho_{\lambda} B_{1}-D_{1}, \sigma, \sigma\right)^{t}, \\
\widetilde{r}_{2 \lambda} & =\frac{1}{2 \rho_{\lambda}^{2} \sigma}\left(\rho_{\lambda} D_{1}+B_{1},-\rho_{\lambda} D_{1}+B_{1}, \rho_{\lambda} B_{1}-D_{1},-\left(\rho_{\lambda} B_{1}+D_{1}\right), \sigma,-\sigma\right)^{t}, \\
\widetilde{r}_{3 \lambda} & =\left(\varepsilon B_{1},-\varepsilon B_{1},-\varepsilon D_{1}, \varepsilon D_{1}, 1,1\right)^{t}, \\
\widetilde{r}_{4 \lambda} & =\left(-\varepsilon B_{1},-\varepsilon B_{1}, \varepsilon D_{1}, \varepsilon D_{1}, 1,-1\right)^{t}, \\
\widetilde{r}_{5 \lambda} & =\frac{1}{2 \rho_{\lambda}^{2} \sigma}\left(\rho_{\lambda} D_{1}+B_{1}, \rho_{\lambda} D_{1}-B_{1}, \rho_{\lambda} B_{1}-D_{1}, \rho_{\lambda} B_{1}+D_{1},-\sigma,-\sigma\right)^{t}, \\
\widetilde{r}_{6 \lambda} & =\frac{1}{2 \rho_{\lambda}^{2} \sigma}\left(\rho_{\lambda} D_{1}-B_{1},-\left(\rho_{\lambda} D_{1}+B_{1}\right), \rho_{\lambda} B_{1}+D_{1},-\rho_{\lambda} B_{1}+D_{1},-\sigma, \sigma\right)^{t} .
\end{aligned}\right.
$$

In this case, the constant sequences $\left(\widetilde{l}_{i \lambda}\right)_{\lambda}$ and $\left(\widetilde{r}_{i \lambda}\right)_{\lambda}$ are bounded and $\widetilde{l}_{i \lambda} \widetilde{r}_{j \lambda}=\delta_{i j}$ for all $1 \leq i, j \leq 6$. On the other hand, when $\sigma=0$, we have $B_{1}=D_{1}=0$, and the matrix $\widetilde{A}_{\lambda}$ is symmetric and independent of $\lambda$. Then we may choose arbitrarily its left and right eigenvectors $\widetilde{l}_{i}$ and $\widetilde{r}_{j}$ such that $\widetilde{l}_{i} \widetilde{r}_{j}=\delta_{i j}$ for all $1 \leq i, j \leq 6$.

Using the explicit formula (3.16), we have

$$
\widetilde{v}_{\lambda}(t, x)=\sum_{i=1}^{6}\left(\widetilde{l}_{i \lambda} \widetilde{v}_{\lambda}^{0}\right)\left(X_{\lambda}^{0}\left(Y_{\lambda}(t, x)-\nu_{i} t\right)\right) \widetilde{r}_{i \lambda},
$$

where

$$
\widetilde{v}_{\lambda}^{0}=\left(v_{3 \lambda}^{0}, \cdots, v_{8 \lambda}^{0}\right)^{t}, \quad X_{\lambda}^{0}=\left(Y_{\lambda}^{0}\right)^{-1}, \quad Y_{\lambda}(t, \cdot)=X_{\lambda}^{-1}(t, \cdot)
$$

with

$$
\begin{gathered}
Y_{\lambda}^{0}(x)=\int_{0}^{x} h_{\lambda}^{0}(\xi) d \xi \\
X_{\lambda}(t, y)=\frac{1}{2} \int_{0}^{y+t}\left(v_{2 \lambda}^{0}+\frac{\rho_{\lambda}}{h_{\lambda}^{0}}\right)\left(X_{\lambda}^{0}(\xi)\right) d \xi-\frac{1}{2} \int_{0}^{y-t}\left(v_{2 \lambda}^{0}-\frac{\rho_{\lambda}}{h_{\lambda}^{0}}\right)\left(X_{\lambda}^{0}(\xi)\right) d \xi
\end{gathered}
$$

Since the sequence $\left(\widetilde{v}_{\lambda}^{0}\right)_{\lambda}$ is assumed to be bounded in $L^{\infty}(\mathbb{R})$, we deduce that $\left(\widetilde{v}_{\lambda}\right)_{\lambda}$ is bounded in $L^{\infty}\left(\mathbb{R}^{+} \times \mathbb{R}\right)$. This proves Lemma 4.2 together with the definition (4.2) and $h_{\lambda}=1+O(\varepsilon)$.

4.2. Convergence. From Lemmas 4.1-4.2, we have

$$
h_{\lambda} \longrightarrow 1, \quad \text { in } L^{\infty}\left(\mathbb{R}^{+} \times \mathbb{R}\right) \text { strongly, }
$$

and up to subsequences (not relabeled),

$$
\left(D_{2 \lambda}, D_{3 \lambda}, B_{2 \lambda}, B_{3 \lambda}, P_{2 \lambda}, P_{3 \lambda}\right) \longrightarrow\left(D_{2}, D_{3}, B_{2}, B_{3}, P_{2}, P_{3}\right),
$$

in $L^{\infty}\left(\mathbb{R}^{+} \times \mathbb{R}\right)$ weakly-*. Recall $\varepsilon=\lambda^{2(\alpha-1)} \rightarrow 0$ as $\lambda \rightarrow \infty$. Since the strong convergence of $h_{\lambda}$ is involved in all its nonlinear terms, it is easy to pass to the limit in the system (4.1) in the sense of distributions. The weak limit $\left(D_{2}, D_{3}, B_{2}, B_{3}\right)$ satisfies the linear Maxwell equations :

$$
\left\{\begin{array}{l}
\partial_{t} D_{2}+\partial_{x} B_{3}=0 \\
\partial_{t} D_{3}-\partial_{x} B_{2}=0 \\
\partial_{t} B_{2}-\partial_{x} D_{3}=0 \\
\partial_{t} B_{3}+\partial_{x} D_{2}=0
\end{array}\right.
$$


and $\left(P_{2}, P_{3}\right)$ satisfies

$$
\left\{\begin{array}{l}
\partial_{t} P_{2}-\partial_{x}\left(D_{1} D_{2}+B_{1} B_{2}\right)=0, \\
\partial_{t} P_{3}-\partial_{x}\left(D_{1} D_{3}+B_{1} B_{3}\right)=0 .
\end{array}\right.
$$

Now consider the limit of $h_{1 \lambda}=\left(h_{\lambda}-1\right) / \varepsilon$ and $P_{1 \lambda}$. Using (4.1), we have

$$
\left\{\begin{array}{l}
\partial_{t} h_{1 \lambda}+\partial_{x} P_{1 \lambda}=0, \\
\partial_{t} P_{1 \lambda}+\partial_{x}\left(\frac{\varepsilon^{2} P_{1 \lambda}-\rho_{\lambda}^{2}}{\varepsilon\left(1+\varepsilon h_{1 \lambda}\right)}\right)=0 .
\end{array}\right.
$$

From Lemma 4.1, the sequences $\left(h_{1 \lambda}\right)_{\lambda}$ and $\left(P_{1 \lambda}\right)_{\lambda}$ are bounded in $L^{\infty}\left(\mathbb{R}^{+} \times \mathbb{R}\right)$. Then

$$
\frac{\varepsilon^{2} P_{1 \lambda}}{\varepsilon\left(1+\varepsilon h_{1 \lambda}\right)} \longrightarrow 0 \quad \text { in } L^{\infty}\left(\mathbb{R}^{+} \times \mathbb{R}\right) \text { strongly, }
$$

and up to subsequences,

$$
h_{1 \lambda} \longrightarrow h_{1}, \quad P_{1 \lambda} \longrightarrow P_{1}, \quad \text { in } L^{\infty}\left(\mathbb{R}^{+} \times \mathbb{R}\right) \text { weakly- } * .
$$

Since

we obtain

$$
\rho_{\lambda}^{2}=1+\varepsilon \sigma, \quad \frac{1}{1+\varepsilon h_{1 \lambda}}=1-\varepsilon h_{1 \lambda}+O\left(\varepsilon^{2}\right)
$$

$$
-\partial_{x}\left(\frac{\rho_{\lambda}^{2}}{\varepsilon\left(1+\varepsilon h_{1 \lambda}\right)}\right)=\partial_{x} h_{1 \lambda}+e_{\lambda},
$$

with $\lim _{\lambda \rightarrow \infty} e_{\lambda}=0$ in $L^{\infty}\left(\mathbb{R}^{+} ; W^{-1, \infty}(\mathbb{R})\right)$. Obviously, we can pass to the limit in (4.9) in the sense of distributions to obtain the linear equations :

$$
\left\{\begin{array}{l}
\partial_{t} h_{1}+\partial_{x} P_{1}=0 \\
\partial_{t} P_{1}+\partial_{x} h_{1}=0 .
\end{array}\right.
$$

On the other hand, the sequences $\left(h_{1 \lambda}^{0}\right)_{\lambda}$ and $\left(v_{i \lambda}^{0}\right)_{\lambda}(2 \leq i \leq 8)$ being bounded in $L^{\infty}(\mathbb{R})$, then up to subsequences, we have

$$
h_{1 \lambda}^{0} \longrightarrow h_{1}^{0}, \quad v_{i \lambda}^{0} \longrightarrow v_{i}^{0}, \quad \text { in } L^{\infty}(\mathbb{R}) \text { weakly-*, }
$$

with $\left(h_{1}^{0}, v_{2}^{0}, \cdots, v_{8}^{0}\right) \in L^{\infty}(\mathbb{R})$. Together with (4.3) for the expression of $h_{\lambda}^{0}$, they imply that

$$
\left(P_{1 \lambda}^{0}, D_{2 \lambda}^{0}, D_{3 \lambda}^{0}, B_{2 \lambda}^{0}, B_{3 \lambda}^{0}, P_{2 \lambda}^{0}, P_{3 \lambda}^{0}\right) \longrightarrow\left(v_{2}^{0}, \cdots, v_{8}^{0}\right), \quad \text { in } L^{\infty}(\mathbb{R}) \text { weakly- * . }
$$

It is clear that the linear system (4.7)-(4.8) and (4.11) is hyperbolic. In particular, (4.7)(4.8) imply that

$$
\partial_{t}\left(P_{2}+D_{1} B_{3}-B_{1} D_{3}\right)=\partial_{t}\left(P_{3}+B_{1} D_{2}-D_{1} B_{2}\right)=0
$$

which is in accordance with $P=D \times B$. Then its Cauchy problem with the initial conditions :

$$
t=0: \quad\left(h_{1}, P_{1}, D_{2}, D_{3}, B_{2}, B_{3}, P_{2}, P_{3}\right)=\left(h_{1}^{0}, v_{2}^{0}, \cdots, v_{8}^{0}\right)(x), \quad x \in \mathbb{R}
$$

admits a unique solution $\left(h_{1}, P_{1}, D_{2}, D_{3}, B_{2}, B_{3}, P_{2}, P_{3}\right) \in L^{\infty}\left(\mathbb{R}^{+} \times \mathbb{R}\right)$. This implies the convergence of the whole sequence. 
Theorem 4.1. Under the assumptions of Lemma 4.1, as $\lambda \rightarrow \infty$, the solution of the Cauchy problem (4.1) and (4.3) is convergent in the sense of (4.5)-(4.6), (4.10) and (4.12), where the limit $\left(h_{1}, P_{1}, D_{2}, D_{3}, B_{2}, B_{3}, P_{2}, P_{3}\right) \in L^{\infty}\left(\mathbb{R}^{+} \times \mathbb{R}\right)$ is the unique solution of the problem (4.7)-(4.8), (4.11) and (4.13).

\section{Limit TOWARD PMHD EQUations in ONE DIMENSION}

In this last section, we justify the limit $\lambda \rightarrow 0$ in the $\lambda$-ABI system (3.2) with the initial conditions :

$$
t=0: \quad h=h_{\lambda}^{0}(x), \quad v_{i}=v_{i \lambda}^{0}(x) \quad(2 \leq i \leq 8), \quad x \in \mathbb{R} .
$$

Similarly to (3.10), we assume that the sequences of the initial data satisfy :

(H1) $\left(h_{\lambda}^{0}\right)_{\lambda}$ and $\left(v_{i \lambda}^{0}\right)_{\lambda}(2 \leq i \leq 8)$ are bounded in $L^{\infty}(\mathbb{R})$,

(H2) $\inf _{x \in \mathbb{R}}\left(v_{2 \lambda}^{0}(x)+\frac{a_{\lambda}}{h_{\lambda}^{0}(x)}\right) \geq b_{2}>b_{1} \geq \sup _{x \in \mathbb{R}}\left(v_{2 \lambda}^{0}(x)-\frac{a_{\lambda}}{h_{\lambda}^{0}(x)}\right)$,

where $b_{1}$ and $b_{2}$ are two constants independent of $\lambda$,

$$
a_{\lambda}=\sqrt{\lambda^{2}+B_{1}^{2}+\lambda^{2} D_{1}^{2}} .
$$

By Proposition 3.1, to guarantee the hyperbolicity of the $\lambda$-ABI system for all $\lambda \geq 0$, we require $a_{\lambda} \neq 0$. Therefore, we assume here $B_{1} \neq 0$.

Applying Proposition 3.2 to the Cauchy problem (3.2) and (5.1), we obtain a unique entropy solution $v_{\lambda}=\left(h_{\lambda}^{-1}, v_{2 \lambda}, \cdots, v_{8 \lambda}\right)^{t} \in L^{\infty}\left(\mathbb{R}^{+} \times \mathbb{R}\right)$. This solution satisfies the maximum principle (3.14)-(3.15). Together with the assumption (H2), we deduce that for sufficiently small $\lambda>0$,

$$
0<\underline{h} \leq h_{\lambda}(t, x) \leq \bar{h}, \quad \text { a.e. }(t, x) \in \mathbb{R}^{+} \times \mathbb{R},
$$

where $\underline{h}$ and $\bar{h}$ are two constants independent of $\lambda$. From (3.16), $v_{\lambda}$ can be expressed as :

$$
v_{\lambda}(t, x)=\sum_{i=1}^{8}\left(l_{i \lambda} v_{\lambda}^{0}\right)\left(X_{\lambda}^{0}\left(Y_{\lambda}(t, x)-\widetilde{\mu}_{i} t\right)\right) r_{i \lambda},
$$

where $\widetilde{\mu}_{i}, l_{i \lambda}$ and $r_{i \lambda}$ are defined in (3.5)-(3.7), respectively, $X_{\lambda}^{0}$ and $Y_{\lambda}$ are given by the formulas (3.11)-(3.13) in which $\left(h^{0}, v_{2}^{0}\right)$ are replaced by $\left(h_{\lambda}^{0}, v_{2 \lambda}^{0}\right)$.

Since $a_{\lambda}=\left|B_{1}\right|+O\left(\lambda^{2}\right)$ and $\beta=B_{1}^{2}+\lambda^{2} D_{1}^{2}$, from (3.6)-(3.7), it is easy to see that $\left(l_{i \lambda}\right)_{\lambda}$ and $\left(r_{i \lambda}\right)_{\lambda}$ are bounded sequences. We conclude from (5.2)-(5.3) that the sequence $U_{\lambda}=\left(h_{\lambda}, P_{1 \lambda}, D_{2 \lambda}, D_{3 \lambda}, B_{2 \lambda}, B_{3 \lambda}, P_{2 \lambda}, P_{3 \lambda}\right)$ is bounded in $L^{\infty}\left(\mathbb{R}^{+} \times \mathbb{R}\right)$. Then, up to subsequences, we have

$$
U_{\lambda} \longrightarrow\left(h, P_{1}, D_{2}, D_{3}, B_{2}, B_{3}, P_{2}, P_{3}\right), \quad \text { in } L^{\infty}\left(\mathbb{R}^{+} \times \mathbb{R}\right) \text { weakly- } * .
$$

In order to pass to the limit in the nonlinear terms of (3.2), we proceed as in [17] using compensated compactness arguments. For instance, let

$$
V_{\lambda}=\left(h_{\lambda}, P_{1 \lambda}\right)^{t}, \quad W_{\lambda}=\left(\frac{P_{1 \lambda}^{2}-a_{\lambda}^{2}}{h_{\lambda}},-P_{1 \lambda}\right)^{t}
$$

Then $\left(V_{\lambda}\right)_{\lambda}$ and $\left(W_{\lambda}\right)_{\lambda}$ are two bounded sequences in $L^{\infty}\left(\mathbb{R}^{+} \times \mathbb{R}\right)$ then in $L_{\text {loc }}^{2}\left(\mathbb{R}^{+} \times \mathbb{R}\right)$. Moreover, they satisfy

$$
\operatorname{div}_{t, x} V_{\lambda}=0, \quad \operatorname{rot}_{t, x} W_{\lambda}=0 .
$$


Hence, by Tartar equation [21] and up to subsequences, we have, in the sense of distributions

$$
\lim _{\lambda \rightarrow 0} V_{\lambda} \cdot W_{\lambda}=\lim _{\lambda \rightarrow 0} V_{\lambda} \cdot \lim _{\lambda \rightarrow 0} W_{\lambda}
$$

i.e.

$$
-\lim _{\lambda \rightarrow 0} a_{\lambda}^{2}=h \chi-P_{1}^{2}
$$

where $\chi$ is the weak limit of the sequence $\left(\left(P_{1 \lambda}^{2}-a_{\lambda}^{2}\right) / h_{\lambda}\right)_{\lambda}$. Since $\lim _{\lambda \rightarrow 0} a_{\lambda}^{2}=-B_{1}^{2}$, we obtain

$$
\chi=\frac{P_{1}^{2}-B_{1}^{2}}{h} .
$$

Thus, $\left(h, P_{1}\right)$ is a solution of the system :

$$
\left\{\begin{array}{l}
\partial_{t} h+\partial_{x} P_{1}=0 \\
\partial_{t} P_{1}+\partial_{x}\left(\frac{P_{1}^{2}-B_{1}^{2}}{h}\right)=0 .
\end{array}\right.
$$

Similarly, using the first and the rest equations in the $\lambda$-ABI system (3.2) and the compensated compactness arguments, we can pass to the limit in (3.2) in the sense of distributions to obtain :

$$
\left\{\begin{array}{l}
\partial_{t} D_{2}+\partial_{x}\left(\frac{B_{3}+D_{2} P_{1}-D_{1} P_{2}}{h}\right)=0, \\
\partial_{t} D_{3}+\partial_{x}\left(\frac{-B_{2}+D_{3} P_{1}-D_{1} P_{3}}{h}\right)=0, \\
\partial_{t} B_{2}+\partial_{x}\left(\frac{B_{2} P_{1}-B_{1} P_{2}}{h}\right)=0, \\
\partial_{t} B_{3}+\partial_{x}\left(\frac{B_{3} P_{1}-B_{1} P_{3}}{h}\right)=0, \\
\partial_{t} P_{2}+\partial_{x}\left(\frac{P_{1} P_{2}-B_{1} B_{2}}{h}\right)=0, \\
\partial_{t} P_{3}+\partial_{x}\left(\frac{P_{1} P_{3}-B_{1} B_{3}}{h}\right)=0 .
\end{array}\right.
$$

Furthermore, as $\lambda \rightarrow 0$, we have obviously (up to subsequences),

$$
\left(h_{\lambda}^{0}, P_{1 \lambda}^{0}, D_{2 \lambda}^{0}, D_{3 \lambda}^{0}, B_{2 \lambda}^{0}, B_{3 \lambda}^{0}, P_{2 \lambda}^{0}, P_{3 \lambda}^{0}\right) \longrightarrow\left(h^{0}, P_{1}^{0}, D_{2}^{0}, D_{3}^{0}, B_{2}^{0}, B_{3}^{0}, P_{2}^{0}, P_{3}^{0}\right),
$$

in $L^{\infty}(\mathbb{R})$ weakly-*, with $\left(h^{0}, P_{1}^{0}, D_{2}^{0}, D_{3}^{0}, B_{2}^{0}, B_{3}^{0}, P_{2}^{0}, P_{3}^{0}\right) \in L^{\infty}(\mathbb{R})$. From Proposition 3.1 with $B_{1} \neq 0$, we know that the system (5.4)-(5.5) is a linear Lagrangian one and hyperbolic. Then its Cauchy problem with the initial conditions :

$$
t=0:\left(h, P_{1}, D_{2}, D_{3}, B_{2}, B_{3}, P_{2}, P_{3}\right)=\left(h^{0}, P_{1}^{0}, D_{2}^{0}, D_{3}^{0}, B_{2}^{0}, B_{3}^{0}, P_{2}^{0}, P_{3}^{0}\right)(x), x \in \mathbb{R}
$$

admits a unique entropy solution $\left(h, P_{1}, D_{2}, D_{3}, B_{2}, B_{3}, P_{2}, P_{3}\right) \in L^{\infty}\left(\mathbb{R}^{+} \times \mathbb{R}\right)$. This implies the convergence of the whole sequence.

Theorem 5.1. Let the assumptions (H1)-(H2) hold and $B_{1} \neq 0$. Then, as $\lambda \rightarrow 0$, the solution of the Cauchy problem (3.2) and (5.1) is convergent in the sense of $L^{\infty}\left(\mathbb{R}^{+} \times \mathbb{R}\right)$ weakly-*, and the limit $\left(h, P_{1}, D_{2}, D_{3}, B_{2}, B_{3}, P_{2}, P_{3}\right) \in L^{\infty}\left(\mathbb{R}^{+} \times \mathbb{R}\right)$ is the unique entropy solution of the Cauchy problem (5.4)-(5.5) and (5.6). 


\section{REFERENCES}

[1] G. Boillat, Non linear hyperbolic fields and waves, Lecture Notes Math., Vol. 1640, 1-47, SpringerVerlag, 1996.

[2] G. Boillat, Euler's variational equations with independent momentum, Il Nuovo Cimento, 119 B (2005), 839-847.

[3] G. Boillat and T. Ruggeri, Energy momentum, wave velocities and characteristic shocks in Euler's variational equations with application to the Born-Infeld theory, J. Math. Phys. 45 (2004), 3468-3478.

[4] M. Born and L. Infeld, Foundation of the new field theory, Proc. Roy. Soc. London, A144 (1934), 425-451.

[5] Y. Brenier, Hydrodynamic structure of the augmented Born-Infeld equations, Arch. Rat. Mech. Anal. 172 (2004), 65-91.

[6] Y. Brenier, A note on deformations of 2D fluid motions using 3D Born-Infeld equations, Monatsh. Math. 142 (2004), 113-122.

[7] Y. Brenier, Order preserving vibrating strings and applications to Electrodynamics and Magnetohydrodynamics, Methods Appl. Anal. 11 (2004), 515-532.

[8] Y. Brenier and W.A. Yong, Derivation of particle, string, and membrane motions from the Born-Infeld electromagnetism, J. Math. Phys. 46 (2005), 062305.

[9] A. Bressan, Hyperbolic Systems of Conservation Laws : The One Dimensional Cauchy Problem, Oxford Lecture Series in Math. and its Applications, Vol. 20, 2000.

[10] D. Chae and H. Huh, Global existence for small initial data in the Born-Infeld equations, J. Math. Phys. 44 (2003), 6132-6139.

[11] C. Dafermos, Hyperbolic Conservation Laws in Continuum Physics, Grundlehren der Mathematischen Wissenschaften, 325, Springer-Verlag, 2000.

[12] G.W. Gibbons, Aspects of Born-Infeld theory and string / M-theory, hep-th / 0106059.

[13] G.W. Gibbons and C.A.R. Herdeiro, Born-Infeld theory and stringy causality, Phys. Rev. D63 (2001), 064006.

[14] Ph. LeFloch, Hyperbolic Systems of Conservation Laws, Lectures in Math. Birkhäuser, 2002.

[15] Y.J. Peng, Explicit solutions for $2 \times 2$ linearly degenerate systems, Appl. Math. Letters, 11 (1998), 75-78.

[16] Y.J. Peng, Entropy solutions of Born-Infeld systems in one space dimension, Rend. Circ. Mat. Palermo. Serie II, 78 (2006), 259-271.

[17] Y.J. Peng, Euler-Lagrange change of variables in conservation laws, Nonlinearity, 20 (2007), 19271953.

[18] D. Serre, Un système hyperbolique non linéaire avec des données oscillantes, C.R. Acad. Sci. Paris, Série I, t. 302, no 3 (1986), 115-118.

[19] D. Serre, Systèmes de Lois de Conservation, Diderot, Paris, 1996.

[20] D. Serre, Hyperbolicity of the nonlinear models of Maxwell's equations, Arch. Rat. Mech. Anal. 172 (2004), 309-331.

[21] L. Tartar, Compensated compactness and applications to partial differential equations, Nonlinear analysis and mechanics : Heriot-Watt symposium, Vol. IV, 136-212, Research Notes in Math., 39, Pitman, 1979.

[22] D. Wagner, Equivalence of the Euler and Lagrangian equations of gas dynamics for weak solutions, J. Diff. Eqns. 68 (1987), 118-136. 